\title{
Breastfeeding and maternal HIV-1 disease progression and mortality
}

\section{Citation}

Sedgh, Gilda, Donna Spiegelman, Ulla Larsen, Gernard Msamanga, and Wafaie W Fawzi. 2004. "Breastfeeding and Maternal HIV-1 Disease Progression and Mortality." AIDS 18 (7): 1043-49. https://doi.org/10.1097/00002030-200404300-00013.

\section{Permanent link}

http://nrs.harvard.edu/urn-3:HUL.InstRepos:41384723

\section{Terms of Use}

This article was downloaded from Harvard University's DASH repository, and is made available under the terms and conditions applicable to Other Posted Material, as set forth at http:// nrs.harvard.edu/urn-3:HUL.InstRepos:dash.current.terms-of-use\#LAA

\section{Share Your Story}

The Harvard community has made this article openly available.

Please share how this access benefits you. Submit a story.

Accessibility 


\title{
Breastfeeding and maternal HIV-1 disease progression and mortality
}

\author{
AIDS: Volume 18(7) 30 April 2004 pp 1043-1049
}

Sedgh, Gildaa; Spiegelman, Donnaa,c; Larsen, Ullab; Msamanga, Gernarde; Fawzi, Wafaie Wa, d

From the Departments of aEpidemiology, bPopulation and International Health, cBiostatistics and dNutrition, Harvard School of Public Health, Boston, Massachusetts, USA and the eDepartment of Community Health, Muhimbili University College of Health

Sciences, Dar es Salaam, Tanzania.Correspondence to Wafaie W. Fawzi, Department of Nutrition, Harvard School of Public Health, 665 Huntington Avenue, Boston, MA 02115, USA.Tel: +1617432 2086; fax: +1 617432 2435; e-mail: mina@hsph.harvard.edu

\section{Abstract}

Objective: To examine the association between breastfeeding and disease progression among HIV-infected women in Dar es Salaam, Tanzania.Design and methods: Cohort study design with Cox proportional hazards models. Results: The relative risk of death comparing women who recently had been breastfeeding to those who were not breastfeeding was 0.47 (95\% confidence interval, $0.18-1.20$ ). Neither breastfeeding status nor the duration of exclusive or partial breastfeeding was associated with HIV-1 disease progression, represented by death or development of a low CD4 cell count, anemia or excessive weight loss, in multivariate analyses. These associations remained insignificant when women with relatively low and high CD4 cell counts were analyzed separately.Conclusion: There is insufficient evidence to support the hypothesis that breastfeeding is detrimental to the health of HIV-infected women.

\section{Introduction}

Until recently, the question of whether HIV-1 positive mothers should breastfeed their children has focused entirely on the benefits and risks to the infant. Research has been directed at determining whether the risk of vertical transmission of HIV infection outweighs the health and nutritional benefits to the child as a result of breastfeeding.

Recently, investigators have started to question whether breastfeeding can affect the health of HIV-1 positive women. To date, two studies have examined the association between breastfeeding and women's health. A randomized clinical trial of breastfed and formula-fed infants in Nairobi, Kenya, which was designed to study the effects on infant health, found that breastfeeding was associated with increased maternal mortality [1]. By contrast, an observational study of breastfeeding and adverse outcomes among infected women in South Africa yielded mixed and mostly insignificant results [2]. Although these studies represent contributions to this research question, both faced limitations. As the investigators of the randomized trial have noted, the women randomized to breastfeeding and formula feeding may not have been comparable with respect to baseline viral load or frequency of contact with health care providers during follow-up. The observational study observed few of the events of interest and had limited power to observe a significant association or control for potential confounders. Neither study assessed whether there was a dose-response relationship between the duration of breastfeeding and maternal disease progression or whether the association was dependent on the mother's baseline health status.

If an association between breastfeeding and the health of HIV-1-positive women existed, it could have important implications for programs and policies relating to breastfeeding, and to the decision-making processes of HIV-infected women. 
This study examines the association between breastfeeding and maternal HIV-1 disease progression by assessing whether breastfeeding is associated with time to death, low CD4 cell count, anemia and weight loss during follow-up in a cohort of HIV-infected women in Dar es Salaam, Tanzania.

\section{Methods}

The data for these analyses were collected for a randomized clinical trial aimed at studying the effects of vitamin supplementation on HIV-1 disease progression and vertical transmission[3]. Women were recruited in antenatal clinics between April 1995 and July 1997. Eligible HIV-1-positive women were pregnant, at 12-27 weeks gestation, residing in Dar es Salaam, and intending to stay in the city for at least 1 year.

Investigators conducted clinical exams and collected socio-demographic information including women's age, education, marital status and birth histories upon enrollment into the study. Laboratory blood tests including T-lymphocyte and hemoglobin counts were conducted upon enrollment and repeated semi- annually.

Women were scheduled for monthly visits with a physician and a nurse from the study team; in December 1997, the protocol was revised to call for visits with a physician every third month. In the months when a woman was checked by both a physician and a nurse, we were able to reassess the woman's clinical stage of disease using a modified version of the World Health Organization's recommended staging system [4]. When a woman missed a visit to the study clinic, a member of the study team visited her home to follow-up and ascertain her vital status.

Women who delivered live-born children were scheduled to bring their infants for monthly visits with a study nurse. The nurse examined the child and collected information on whether the child had breastfed in the preceding month, and when the child was started on other foods or sources of milk in addition to breast milk.

The Wilcoxon and Kruskal-Wallis rank sum tests were used to test the difference in mean breastfeeding duration by socio-demographic and health-related characteristics of the women. A prospective cohort study design and multivariate Cox proportional hazards models were used to examine whether breastfeeding was associated with a more rapid progression to death. We also examined whether breastfeeding was associated with other known markers of disease progression, namely a decline in CD4 cell count below $200 ¥$ 106 cells/I [4]; anemia, indicated here as a drop in hemoglobin levels below $8.5 \mathrm{~g} / \mathrm{dl}$ [5]; and the loss of more than $10 \%$ in body weight [4]. A woman's weight loss during followup was measured against her first weight measurement between 6 and 9 months postpartum. We also examined whether the associations of interest varied according to a woman's baseline CD4 cell count, using the likelihood ratio test to test for interaction. At each observation time, breastfeeding status was defined as whether or not the woman had been breastfeeding exactly 6 months ( 182 days) prior to the time of observation. We also analyzed whether the amount of time a woman had breastfed, defined as the proportion of months she had breastfed up to 6 months or more prior to the month of observation, was associated with progression. As mother's illness has been cited as a common reason for the termination of breastfeeding [6], we considered breastfeeding that preceded the incidence of the adverse outcome to ensure that the observed exposure status was not determined by the development of the adverse outcome. These analyses were repeated with exposure set to precede the outcome by 3,9 and 12 months.

Since the frequency and intensity of breastfeeding are generally greatest when a woman is exclusively breastfeeding, the association of exclusive breastfeeding with subsequent disease progression and death was also explored. A woman was considered to be exclusively breastfeeding if she had not yet introduced any solids or milk from other sources into the child's diet. We could not ascertain when women introduced water or other liquids to their infants' diets; therefore, this definition of exclusive breastfeeding 
encompasses the World Health Organization definition of exclusive breastfeeding (a diet of breast milk with no other liquids or solids except vitamins, mineral supplements, or medicines) and predominant breastfeeding (a diet consistently primarily of breast milk but also including water-based drinks, fruit juice, or other non-nutritional fluids) [7]. These models included women who survived without experiencing the event until all exclusive breastfeeding in the population was complete, and examined whether the total duration of exclusive breastfeeding was associated with adverse health outcomes in the first 2 years after delivery.

In addition to Cox proportional hazards models, marginal structural models were used to control for time-dependent confounders that may both affect and be affected by exposure - namely the woman's recent stage of disease and the survival status of the child - along with other potential confounders of the associations of interest [8].

In all of these analyses, follow-up for each participant ended when she experienced the outcome of interest, was last seen by a member of the study team, or at the end of follow-up, whichever came first. For the purpose of these analyses, follow-up ended on 30 September 2001. Cross-tabulations and the Pearson's c2 test were used to assess whether the women with incomplete follow-up data differed from women with complete follow-up data. The missing indicator method was used to retain observations with missing covariate values in multivariate models [9].

Of 1075 HIV-infected, pregnant women originally randomized, six died before delivery, and the date of delivery was unavailable for 27 women. Ninety-seven women who experienced a fetal loss and 23 women who gave birth to twins were also excluded. Another 96 were lost within 3 months after delivery. Of the 826 eligible women remaining, we were able to ascertain the complete duration of breastfeeding for 597 women, and we have partial information on the duration of breastfeeding for an additional 186 women who were still breastfeeding at their child's last visit to the clinic. The duration of exclusive breastfeeding was established for 698 women.

The study protocol for the randomized trial from which these data were drawn was approved by the Research and Publications Committee of Muhimbili University College of Health Sciences, the Ethical Committee of the National AIDS Control Program of the Tanzanian Ministry of Health, and the Institutional Review Board of the Harvard School of Public Health.

Results

Among the women for whom complete information on breastfeeding duration was available, the mean duration of breastfeeding was 18.2 months (range, 1.0 to 45.2 months). Women with CD4 cell counts below $200 ¥ 106$ cells/l at the start of follow-up breastfed only 14.9 months on average, whereas women with CD4 cell counts of at least $500 ¥ 106$ cells/l breastfed for an average of 18.7 months $(P=0.03)$. Women who were 15-19 years old at the start of follow-up breastfed on average 2.6 months less than women who were 35-39 years old ( 18.0 months versus 20.6 months, $\mathrm{P}=0.02$ ). Women who experienced the death of the breastfeeding child within a month after cessation of breastfeeding breastfed for an average of only 8.8 months, compared with women whose children survived this window of time, who breastfed for 19.4 months on average $(\mathrm{P}<$ 0.0001 ). The data do not indicate whether infants who died shortly after weaning were weaned because they were terminally ill. The mean duration of breastfeeding did not vary significantly by women's baseline clinical stage of disease, educational level, marital status, parity or source of income.

The women who were lost to follow-up in our analysis of mortality outcomes were compared with women who were followed until death or the end of the study period. A woman was considered lost if the study team was unable to establish her vital status during the last 6 months of the follow-up period. Five percent of women were followed for less than a year, and 1-4\% were lost in each subsequent year of follow-up. The women 
who were missing during follow-up tended to be younger $(P=0.05)$ compared with those retained in the study. The two groups of women did not differ with respect to baseline clinical stage of disease, CD4 cell count, education, marital status, source of income or parity.

At each observation time, recent breastfeeding status was defined as whether or not the woman had been breastfeeding 6 months prior to the time of observation. Recent breastfeeding status was not associated with a woman's risk of death in multivariate analysis controlling for other potential risk factors for death, namely her disease stage and CD4 cell count in the month preceding exposure; her baseline age, education and parity; and survival status of the breastfeeding child [relative risk, $0.73 ; 95 \%$ confidence interval $(\mathrm{Cl}), 0.29-1.83$ ]. Breastfeeding was not significantly associated with the risk of anemia, weight loss or decline in CD4 cell count below $200 ¥ 106$ cells/l. These results persisted when we considered breastfeeding that preceded the adverse outcomes by 3,9 or 12 months (data not shown).

These analyses were repeated in subsets of women according to their baseline health status, defined by their CD4 cell counts at baseline. The association of breastfeeding with mortality risk did not vary significantly between women whose baseline CD4 cell counts were below $350 ¥ 106$ cells/I and women with CD4 counts of at least $350 ¥ 106$ cells/I at baseline ( $P$-value, test for interaction $=0.89$ ). Similarly, the relative risks of anemia, weight loss and a decline in CD4 cell count were not modified by baseline CD4 cell counts (results not shown).

When marginal structural models were used to control for the confounding effects of disease progression and child death on breastfeeding status, the association between breastfeeding and maternal death were essentially unchanged and remained insignificant (not shown).

Analyses were also conducted in which the exposure of interest was the total proportion of months a woman had breastfed up to 6 months prior to the month of observation. The relative risk of death for women who had breastfed $50-74 \%$ of the follow-up time, in comparison with women who had breastfed for less than $50 \%$ of the follow-up period, was $0.70(95 \% \mathrm{Cl}, 0.45-1.40)$. The relative mortality risk for women who had breastfed for at least $75 \%$ of the follow-up period was $0.90(95 \% \mathrm{Cl}, 0.41-1.99)$, compared with women who had breastfed less than $50 \%$ of that time (P-value, test for trend $=0.65$ ). The proportion of months a woman had breastfed was not significantly associated with her risk of a low CD4 cell count, anemia or excessive weight loss in multivariate analyses. There were no significant trends in the risk of an adverse outcome with longer durations of breastfeeding.

The average duration of exclusive breastfeeding in this population was 2.9 months (range, 1-5 months). The duration of exclusive breastfeeding during the first 5 months of followup was not associated with subsequent death, low CD4 cell count, anemia or excessive weight loss up to 2 years after delivery. There were no significant trends in the risk of an adverse outcome with longer durations of exclusive breastfeeding.

Tests for proportional hazards indicated that the association between breastfeeding and adverse maternal outcomes did not change over time.Discussion

Neither breastfeeding status nor the duration of breastfeeding was associated with maternal HIV disease progression represented by death, a low CD4 cell count, anemia or excessive weight loss in multivariate analyses in this cohort of women. These outcomes were not associated with the recent breastfeeding status or the duration of exclusive or partial breastfeeding. These findings were robust to variations in the timing of exposure relative to the outcome. The results did not vary significantly when relatively healthy women were analyzed separately from women with low baseline CD4 cell counts. The results were also qualitatively unchanged when marginal structural models were used to control for time-varying confounders that may both determine and be affected by 
breastfeeding status.

It is often difficult to attain adequate power in a cohort study looking at mortality outcomes. The power of this study to observe an association between breastfeeding and death was limited by the number of events observed. Only 109 of the eligible women with breastfeeding data died during follow-up. The cohort study by Coutsoudis et al. [2] observed five deaths and 75 clinical problems during follow-up. Nduati et al. [1] observed 24 maternal deaths during follow-up in the randomized trial. Notably, the associations of interest in our study remained insignificant in analyses of weight loss and anemia, which was experienced by 209 and 255 women, respectively.

As in most cohort studies, this study is vulnerable to loss of subjects during follow-up. In order for this loss to introduce a bias in the estimates of association, the women for whom follow-up data are missing must be different from the women retained in the study with respect to both exposure and the outcome. Although we cannot determine with certainty whether this pattern occurred in our study, analyses of the data available indicated that these two groups of women were similar on most baseline health and socio- demographic characteristics.

Our findings do not support those of the clinical trial reported on by Nduati et al. [1], in which women were randomized to either breastfeed or formula feed their infants from birth. That study indicated that women randomized to breastfeeding faced a higher maternal mortality rate than women who formula fed their infants. The breastfeeding women in the clinical trial had a higher mean plasma HIV-1 viral load at randomization than the formula-feeding group, suggesting a higher baseline risk of disease progression. It has also been noted that formula-feeding mothers might have interacted more frequently with educators and health care providers on the study team in order to ensure proper formula preparation, resulting in a health advantage not directly related to mode of feeding [10].The proportion of women lost to follow-up was similar in both arms of the trial, but the average duration of follow-up was nearly $20 \%$ longer in the breastfeeding group in comparison with the formula-feeding group, allowing more time for the accrual of deaths.

Our results are in close agreement with those of the cohort study by Coutsoudis et al. [2], which did not observe relationships of breastfeeding with the poor health of HIV-infected women. The primary constraint of that study was its limited power. Despite this constraint, breastfeeding did appear significantly protective of the risk of certain infections, and was associated with higher CD4 cell counts and hemoglobin levels in subgroups of the sample. However, those analyses included little or no control for confounding.

Nduati et al suggested that lactation in the setting of HIV-1 infection might place an excessive burden on the mother's energy reserves and exacerbate a woman's risk of maternal depletion syndrome [1]. Various definitions of maternal depletion are found in the literature [11, 12]. The essential elements of this syndrome include short birth intervals and prolonged periods of lactation combined with marginally inadequate diets, resulting in a decline in mother's nutritional status. The possibility that breastfeeding by HIV-1-infected women increases the risk of maternal depletion cannot be ruled out with certainty. However, research suggests that lactation may not deplete a woman's energy reserves to the extent that is often assumed. It has been noted that the association of breastfeeding with post-partum weight loss varies substantially across populations, including those in developing countries, with many studies failing to show an association between breastfeeding and weight loss, and others even showing a slower rate of weight loss among breastfeeding versus formula-feeding women $[12,13]$. Complementing these findings is evidence from physiological studies that metabolic efficiency is increased during lactation, and that the energy demands of breastfeeding are less than often reported [1416]. In particular, neuroendocrine changes during breastfeeding appear to be associated with increased insulin sensitivity [17], which reduces the energy expenditure required for oxidation of fat $[15,16]$. 
While the present study is observational in nature and thus vulnerable to residual confounding, it also had numerous advantages. We looked for a dose-response relationship between breastfeeding duration and disease progression by modeling the duration of total and exclusive breastfeeding. We were able to look for modification of the association of interest by the baseline health status of the mother; we examined multiple endpoints corresponding to the mother's disease progression and also representing potential mechanisms by which breastfeeding might affect mortality risk; and we varied the timing of exposure relative to the outcomes of interest.

Our findings indicate that breastfeeding is not detrimental to the health of HIV-1 infected women. Although additional studies are called for to confirm the results, these findings suggest that HIV-1-positive women should continue to base their decisions about breastfeeding on their assessments of the health and nutritional benefits of breastfeeding, the risks associated with not breastfeeding, and risks of vertical transmission to their children.

References

1. Nduati R, Richardson BA, John G, Mbori-Ngacha D, Mwatha A, Ndinya-Achola J, et al. Effect of breastfeeding on mortality among HIV-1 infected women: a randomised trial. Lancet 2001; 357:1651-1655.

2. Coutsoudis A, Coovadia H, Pillay K, Kuhn L. Are HIV-infected women who breastfeed at increased risk of mortality? AIDS 2001; 15:653-635.

3. Fawzi WW, Msamanga GI, Spiegelman D, Urassa EJ, Hunter DJ. Rationale and design of the Tanzania Vitamin and HIV Infection Trial. Control Clin Trials 1999; 20:75-90.

4. Anon. Proposed 'World Health Organization staging system for HIV infection and disease': preliminary testing by an international collaborative cross-sectional study. The WHO International Collaborating Group for the Study of the WHO Staging System. AIDS 1993; 7:711-718.

5. Fawzi WW, Msamanga GI, Hunter D, Renjifo B, Antelman G, Bang H, et al. Randomized trial of vitamin supplements in relation to transmission of HIV-1 through breastfeeding and early child mortality. AIDS 2002; 16: 1935-1944.

6. Jakobsen MS, Sodemann M, Molbak K, Aaby P. Reason for termination of breastfeeding and the length of breastfeeding. Int J Epidemiol 1996; 25:115-121.

7. World Health Organization. Indicators for Assessing Breastfeeding Practices. WHO/CDD/SER/91.4. Geneva: World Health organization; 1991.

8. Robins J M, Hernan MA, Brumback B. Marginal structural models and causal inference in epidemiology. Epidemiology 2000; 11:550-560.

9. Wilcoxon F. Individual comparisons by ranking methods. Biometrics 1945; 1:80-83.

10. Tompson M. Breastfeeding in HIV-1-positive mothers [correspondence]. Lancet 2001; 358: 1095.

11. Winkvist A, Rasmussen KM, Habicht JP. A new definition of maternal depletion syndrome. Am J Public Health 1992; 82:691-694.

12. Merchant $\mathrm{K}$, Martorell R. Frequent reproductive cycling: does it lead to nutritional depletion of mothers? Prog Food Nutr Sci 1988; 12:339-369.

13. Butte NF, Hopkinson JM, Mehta N, Moon JK, Smith EO. Body composition changes 
during lactation are highly variable among women. J Nutr 1998; 128(Suppl): 381S-385S.

14. Frigerio C, Schutz Y, Prentice A, Whitehead R, J equier E. Is human lactation a particularly efficient process? Eur J Clin Nutr 1991; 45:459-462.

15. Illingworth PJ, Jung RT, Howie PW, Leslie P, Isles TE. Diminution in energy expenditure during lactation. Br Med J (Clin Res Ed) 1986; 292:437-441.

16. Tigas S, Sunehag A, Haymond MW. Metabolic adaptation to feeding and fasting during lactation in humans. J Clin Endocrinol Metab 2002; 87:302-307.

17. Motil K, Thotathuchery M, Montadon CM, Hachey DL, Button TW, Klein PD, et al. Insulin, cortisol and thyroid hormones modulate maternal protein status and milk production and composition in humans. J Nutr 1994; 124: 1248-1257. 